\title{
Temozolomide induces autophagy via ATM-AMPK-ULK1 pathways in glioma
}

\author{
YUHUI ZOU ${ }^{1}$, QIONG WANG ${ }^{2},{\text { BINGLING } \text { LI }^{3}, \text { BING XIE }^{1} \text { and WEIMIN WANG }}^{1}$ \\ Departments of ${ }^{1}$ Neurosurgery, ${ }^{2}$ Medical Information and ${ }^{3}$ Pharmacy, \\ Guangzhou General Hospital of Guangzhou Military Command, Guangzhou, Guangdong 510010, P.R. China
}

Received August 10, 2013; Accepted March 18, 2014

DOI: $10.3892 / \mathrm{mmr} .2014 .2151$

\begin{abstract}
Autophagy is a cytoprotective process, which occurs following temozolomide (TMZ) treatment, and contributes to glioma chemoresistance and TMZ treatment failure. However, the molecular mechanisms by which TMZ induces autophagy are largely unknown. In the current study, the ataxia-telangiectasia mutated (ATM) inhibitor KU-55933, adenosine monophosphate-activated protein kinase (AMPK) inhibitor compound $\mathrm{C}$, and U87MG and U251 cell lines were employed to investigate the molecular mechanisms of TMZ-induced autophagy in glioma, and to evaluate the effects of autophagy inhibition on TMZ cytotoxicity. KU-55933 and compound $\mathrm{C}$ were observed to inhibit the activation of autophagy-initiating kinase ULK1 and result in a significant decrease of autophagy as indicated by depressed LC3B cleavage and acidic vesicular organelle formation. The activation of AMPK-ULK1 was ATM dependent. Autophagy inhibition via the AMPK inhibitor compound $\mathrm{C}$ augmented TMZ cytotoxicity as observed by depressed cell viability, increased $\gamma \mathrm{H} 2 \mathrm{AX}$-marked double-strand breaks (DSBs) and elevated numbers of apoptotic glioma cells. In conclusion, TMZ induced autophagy via ATM-AMPK-ULK1 pathways. TMZ chemoresistance may therefore be overwhelmed by targeting AMPK, particularly for the treatment of $\mathrm{O}^{6}$-methylguanine DNA methyltransferase-negative gliomas.
\end{abstract}

\section{Introduction}

Glioma accounts for $\sim 80 \%$ of primary malignant brain tumors in adults, and progresses rapidly and results in increased rates of mortality compared with any other type of tumor (1). Temozolomide (TMZ), an oral alkylating agent, is the first

Correspondence to: Dr Weimin Wang, Department of Neurosurgery, Guangzhou General Hospital of Guangzhou Military Command, 111 Liuhua Road, Guangzhou, Guangdong 510010, P.R. China E-mail: gzwangwm0@163.com

Key words: autophagy, ataxia-telangiectasia mutated, adenosine monophosphate-activated protein kinase, glioma, temozolomide line chemotherapeutic drug in current standard glioma treatment (2).

The therapeutic benefit of TMZ depends on its ability to alkylate DNA, which often occurs at the N-7 or O-6 position of guanine residues. The minor adduct $\mathrm{O}^{6}$-methylguanine $\left(\mathrm{O}^{6} \mathrm{MeG}\right)$ is the most cytotoxic lesion, which mismatches with thymine. The resulting $\mathrm{O}^{6} \mathrm{MeG} / \mathrm{T}$ mismatches are recognized by the mismatch repair system, which performs futile repair cycles and results in DNA double-strand breaks (DSBs) $(3,4)$. Following DNA damage, ataxia-telangiectasia mutated (ATM), a serine/threonine protein kinase, is recruited to DNA foci, which in turn activates ATM. Activated ATM then transmits the DNA damage signal to downstream substrates and elicits DNA damage responses (5-7).

The emergence of drug resistance often leads to therapeutic failure in the treatment of glioma, precluding long-term survival of the patients. The primary cytotoxic lesion, the $\mathrm{O}^{6} \mathrm{MeG}$ DNA adduct, may be eliminated by $\mathrm{O}^{6}$-methylguanine DNA methyltransferase (MGMT) in gliomas expressing this DNA repair enzyme $(4,8)$. Since the MGMT promoter in almost half of the glioblastoma specimens was methylated and the MGMT promoter methylation status of the primary tumor was retained at recurrence, other chemoresistance mechanisms are critical for TMZ tolerance in MGMT-negative glioblastoma $(9,10)$.

Autophagy, which is characterized by the formation of acidic vesicular organelles (AVOs), is another cellular process critical for glioma cell survival under TMZ treatment. Induction of autophagy by TMZ has been documented in glioma cell lines and surgical specimens, and inhibition of autophagy augments TMZ-induced apoptosis in glioma cells $(11,12)$. However, the molecular mechanism by which TMZ induces autophagy is largely unknown.

In the present study, it was hypothesized that TMZ-induced activation of ATM elicited autophagy. In order to assess this hypothesis, the effect of ATM inhibition on autophagy was evaluated, the activation of AMPK and ULK1 was assessed, which were autophagy-initiating kinases, under ATM inhibition, and their association was investigated.

\section{Materials and methods}

Cell culture and reagents. U87MG and U251 human malignant glioma cell lines were obtained from the Cell Bank of 
the Chinese Academy of Sciences (Shanghai, China). Cells were maintained in Dulbecco's modified Eagle's medium (Gibco-BRL, Carslbad, CA, USA) supplemented with 10\% fetal bovine serum (PAA Laboratories, Pasching, Australia) at $37^{\circ} \mathrm{C}$ in a $5 \% \mathrm{CO}_{2}$-humidified atmosphere. TMZ was supplied by Tasly Pharmaceutical Co., Ltd. (Tianjin, China). ATM kinase inhibitor KU-55933 (sc-202963) and the AMPK inhibitor compound C (P5499) were purchased from Santa Cruz Biotechnology, Inc. (Santa Cruz, CA, USA) and Sigma-Aldrich (St. Louis, MO, USA), respectively. TMZ, KU-55933 and compound $\mathrm{C}$ were dissolved in dimethylsulfoxide (DMSO; Sigma-Aldrich). The final concentration of DMSO in the culture medium did not exceed $0.01 \%$, thus did not effect cell viability or protein expression.

Protein extracts. For experiments using whole-cell lysate samples, cells were washed with ice-cold phosphate-buffered saline (PBS), removed from the culture dishes and incubated in lysis buffer $(150 \mathrm{mM} \mathrm{NaCl}, 0.1 \% \mathrm{NP}-40,0.5 \%$ sodium deoxycholate, $0.1 \%$ SDS, $50 \mathrm{mM}$ Tris, $1 \mathrm{mM}$ DTT, $5 \mathrm{mM}$ $\mathrm{Na}_{3} \mathrm{VO}_{4}, 1 \mathrm{mM}$ phenylmethylsulfonyl fluoride, $10 \mu \mathrm{g} / \mathrm{ml}$ trypsin inhibitor, $10 \mu \mathrm{g} / \mathrm{ml}$ aprotinin and $5 \mu \mathrm{g} / \mathrm{ml}$ leupeptin, $\mathrm{pH}$ 7.4) for $30 \mathrm{~min}$ on ice. The lysate was centrifuged at $12,000 \times \mathrm{g}$ for $30 \mathrm{~min}$ at $4^{\circ} \mathrm{C}$ and the supernatant was collected as a whole cell lysate. For experiments using subcellular fractionation, cells were washed with ice-cold PBS, removed from the culture dishes and incubated in hypotonic protein extract buffer (1 mM EGTA, 1 mM EDTA, $10 \mathrm{mM}$ HEPES, $10 \mathrm{mM} \mathrm{KCl}, 10 \mathrm{mM} \mathrm{NaF}, 1 \mathrm{mM} \mathrm{Na}_{3} \mathrm{VO}_{4}$, $1 \mathrm{mM}$ dithiothreitol, $10 \mathrm{mM} \beta$-glycerophosphate, $100 \mathrm{mg} / \mathrm{ml}$ phenylmethylsulfonyl fluoride and $10 \mathrm{mg} / \mathrm{ml}$ aprotinin) for $10 \mathrm{~min}$ on ice, and lysed by the addition of Igepal CA-630 (final concentration, $0.4 \%$ ) with vigorous mixing for $10 \mathrm{sec}$. The lysate was centrifuged at $12,000 \mathrm{x}$ g for $5 \mathrm{~min}$ at $4^{\circ} \mathrm{C}$ and the supernatant was collected as a cytoplasmic protein extract. The pellet was sonicated using an Ultrasonic homogenizer (BL-96-II L; Voshin Instrument Co., Wuxi, Jiangsu, China) and incubated in hypertonic protein extract buffer (10 mM Tris, $1 \mathrm{mM}$ EGTA, $1 \mathrm{mM}$ EDTA, $400 \mathrm{mM} \mathrm{NaCl}$, $10 \mathrm{mM} \mathrm{NaF}, 1 \mathrm{mM} \mathrm{Na} \mathrm{VO}_{4}, 1 \mathrm{mM}$ dithiothreitol, $10 \mathrm{mM}$ $\beta$-glycerophosphate, $0.5 \%$ Igepal CA-630, $100 \mathrm{mg} / \mathrm{ml}$ phenylmethylsulfonyl fluoride and $10 \mathrm{mg} / \mathrm{ml}$ aprotinin) for $30 \mathrm{~min}$ on ice and centrifuged at $12,000 \mathrm{x} \mathrm{g}$ for $5 \mathrm{~min}$, and the supernatant was collected as a nuclear protein extract. All protein samples were stored at $-80^{\circ} \mathrm{C}$.

Western blot analysis. Western blot analysis was performed using standard methods. $\beta$-actin (4970, Cell Signaling Technology, Inc., Danvers, MA, USA) was used as a loading control. The other primary antibodies used were as follows: Monoclonal anti-phospho-ATM (Ser1981) antibody (5883, Cell Signaling Technology Inc., Danvers, MA, USA), monoclonal anti-ATM antibody (2873, Cell Signaling Technology Inc.), polyclonal anti-AMPK $\alpha$ antibody (2532, Cell Signaling Technology Inc.), monoclonal anti-phospho-AMPK $\alpha$ (Thr172) antibody (2535, Cell Signaling Technology Inc.), polyclonal anti-phosphor-ULK1 (Ser467) antibody (4634, Cell Signaling Technology Inc.), polyclonal anti-LC3B antibody (2775, Cell Signaling), polyclonal anti- $\gamma \mathrm{H} 2 \mathrm{AX}$ antibody (ab2893, Abcam, Cambridge, MA, USA). Microtubule-associated protein light chain 3B (LC3B), which is cleaved into LC3B-I and LC3B-II during autophagy, was used as an autophagy marker (13-15).

Detection of AVOs. Quantification of autophagy by acridine orange staining using flow cytometry was performed as described previously $(16,17)$. Briefly, following drug treatment, acridine orange (sc-358795, Santa Cruz Biotechnology, Inc.) was added at a final concentration of $1 \mu \mathrm{g} / \mathrm{ml}$ for a period of $15 \mathrm{~min}$. All floating and adherent cells were collected, washed with PBS, resuspended with phenol red-free growth medium and analyzed by flow cytofluorometry (guava easyCyte 5HT; Millipore Corporation, Hayward, CA, USA). When excited with a 488-nm laser, the nucleolus of acridine orange-stained cells fluoresced bright green and acidic vesicles emitted bright red fluorescence. The forward scatter threshold was adjusted to omit cellular debris and 5,000 ungated events were analyzed. Cells containing AVOs were identified as double positive cells.

Detection of apoptosis. Cell apoptosis was detected with an Annexin V-fluorescein isothiocyanate (FITC)/propidium iodide (PI) apoptosis detection kit (C1063, Beyotime Biotech., Jiangsu, China) according to the manufacturer's instructions. Briefly, cells were trypsinized with $0.25 \%$ trypsin, washed twice with PBS and collected by centrifugation (192 x g, 5 min). Cells were resuspended with binding buffer at a density of $1 \times 10^{6} / \mathrm{ml}$, stained with Annexin V-FITC and PI for $15 \mathrm{~min}$ in the dark at room temperature and analyzed by flow cytofluorometry (Guava Easycyte 5HT).

Cell viability analysis. MTT assays were performed to assess the sensitivity of cells to drugs. Briefly, glioma cells were seeded at a density of 3,000 cells/well in 96-well microplates. The following day, cells were treated with TMZ and/or compound C for $72 \mathrm{~h}$. Following culture, $20 \mu \mathrm{l}$ MTT $(5 \mathrm{mg} / \mathrm{ml})$ was added to each well, and plates were placed at $37^{\circ} \mathrm{C}$ for $4 \mathrm{~h}$. DMSO $(100 \mu \mathrm{l})$ was added to each well to lyse the cells. Absorbance was measured at $570 \mathrm{~nm}$ using a microplate spectrophotometer (Thermo Scientific Microplate Reader; Thermo Fisher Scientific Inc., Waltham, MA, USA).

Statistical analysis. All experiments were performed in triplicate, and results are presented as the mean \pm standard deviation. Statistical analysis of the data was performed using Student's t-test (for two groups) or one-way analysis of variance (for three or more groups). $\mathrm{P}<0.05$ and $\mathrm{P}<0.01$ were considered to indicate a statistically significant difference.

\section{Results}

TMZ treatment induces autophagy in glioma. Induction of autophagy by TMZ has been documented in glioma cell lines and surgical specimens $(11,12)$. In agreement with this, the current results showed that treatment with $100 \mu \mathrm{M} \mathrm{TMZ}$ induced autophagy as shown by a significant increase of AVOs and enhanced cleavage of LC3B (Fig. 1A and B). ATM, AMPK and ULK1 were activated, as shown by western blot analysis, following TMZ treatment (Fig. 1B).

TMZ induces autophagy via AMPK-ULK1 pathways. It has been documented that AMPK is involved in the 
A
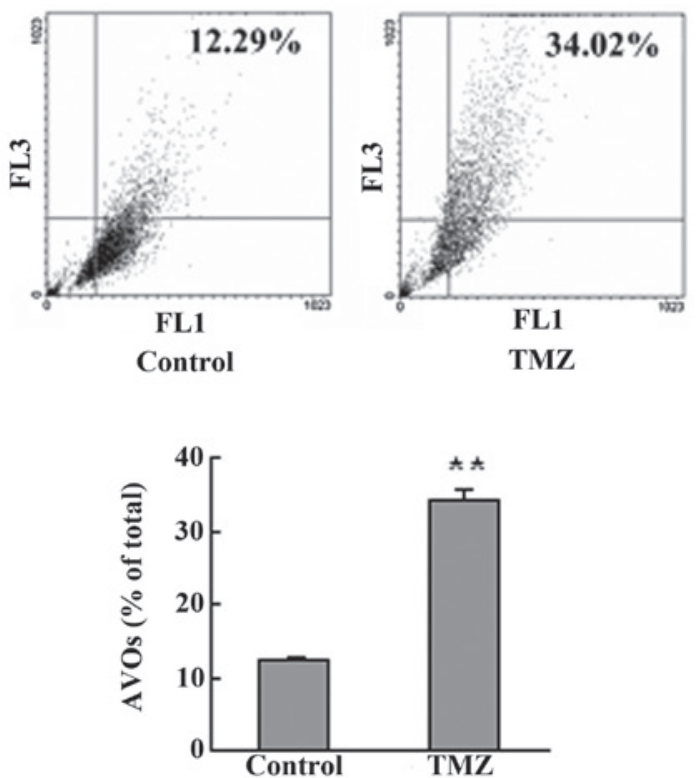

B

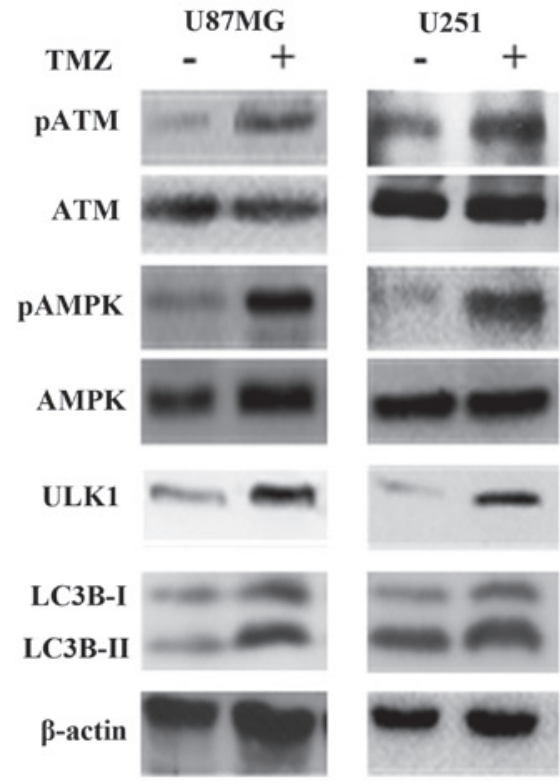

Figure 1. TMZ treatment induced autophagy in glioma. (A) U87MG cells were treated with vehicle or TMZ $(100 \mu \mathrm{M})$ as indicated for $72 \mathrm{~h}$. Following drug treatment, acridine orange was added at a final concentration of $1 \mu \mathrm{g} / \mathrm{ml}$ for a period of $15 \mathrm{~min}$. Next, cells were collected and AVOs were detected by flow cytometry. A total of 5,000 ungated events were analyzed. (B) U87MG and U251 glioma cells were treated with TMZ $(100 \mu \mathrm{M})$ for $72 \mathrm{~h}$. Next, cells were harvested, and analyzed by western blot analysis. Vehicle was used as a negative control. $\beta$-actin was used as a loading control. Data are shown as the mean \pm standard deviation. ${ }^{*} \mathrm{P}<0.05$ and ${ }^{* *} \mathrm{P}<0.01$, vs. control groups, $\mathrm{n}=3$ for each group. TMZ, temozolomide; AVOs, acidic vesicular organelles ATM, ataxia-telangiectasia mutated; AMPK, adenosine monophosphate-activated protein kinase

initiation of autophagosome formation by interacting with mammalian autophagy-initiating kinase ULK1 $(18,19)$. Consistently, the current results showed that inhibition of AMPK with compound C led to depression of ULK1, and that the LC3B cleavage was decreased significantly in the $\mathrm{TMZ}+$ compound $\mathrm{C}$ groups compared with the other groups (Fig. 2). This indicated that TMZ induced autophagy via AMPK-ULK1 pathways.

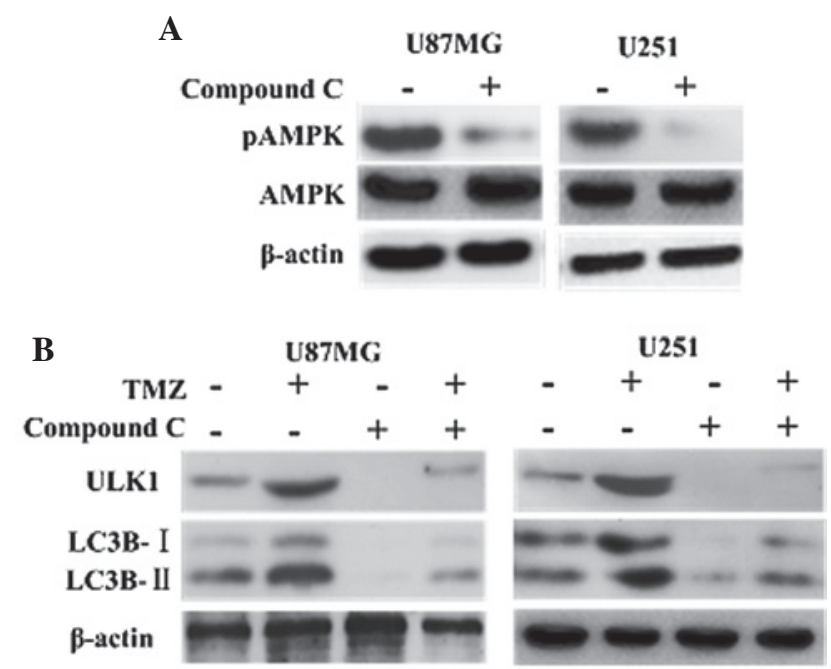

Figure 2. TMZ-induced autophagy via AMPK-ULK1 pathways. (A) U87MG and $\mathrm{U} 251$ cells were treated with $5 \mu \mathrm{M}$ compound $\mathrm{C}$ or vehicle. Following treatment $(72 \mathrm{~h})$, cells were harvested and analyzed by western blot analysis to determine the effect of compound $\mathrm{C}$ on AMPK $\alpha$ (Thr172) phosphorylation. $\beta$-actin was used as a loading control. (B) U87MG and U251 cells were treated with vehicle, TMZ $(100 \mu \mathrm{M})$, compound $\mathrm{C}(5 \mu \mathrm{M})$ or TMZ $(100 \mu \mathrm{M})+$ compound $\mathrm{C}(5 \mu \mathrm{M})$ for $72 \mathrm{~h}$. Next, cells were collected and subjected to western blot analysis to detect ULK1 and cleavage of LC3B. $\beta$-actin was used as a loading control. Experiments were performed in triplicate. TMZ, temozolomide; AMPK, adenosine monophosphate-activated protein kinase.

TMZ-induced autophagy and AMPK-ULK1 activation are ATM dependent. To determine the role of ATM in the autophagy process and AMPK-ULK1 activation, a specific ATM inhibitor, KU-55933U, was used to inhibit ATM phosphorylation and to assess the level of autophagy. Western blot analysis showed that TMZ failed to induce the phosphorylation of AMPK and ULK1 following treatment with $10 \mu \mathrm{M}$ KU-55933, and the expression of LC3B-I and LC3B-II declined significantly compared with the TMZ and control groups (Fig. 3A and B). Flow cytometric analysis showed that inhibition of ATM phosphorylation resulted in fewer AVOs in the TMZ + KU-55933 group compared with the TMZ group (9.24 \pm 0.38 vs. $29.90 \pm 2.14 \%$, respectively, $\mathrm{P}<0.001$; Fig. 3C). The levels of LC3B cleavage and AVOs in the KU-55933 group were also lower compared with the control group (Fig. 3B and C). Therefore, TMZ-induced autophagy and AMPK-ULK1 activation were ATM dependent.

Inhibition of AMPK augmented the cytotoxicity of TMZ by disrupting autophagy. Since autophagy serves as a cytoprotective process and ATM mutation results in ataxia telangiectasia, the AMPK inhibitor compound $\mathrm{C}$ was employed to investigate the effect of autophagy disruption on TMZ cytotoxicity from a possible medical treatment standpoint. AMPK inhibition with compound $\mathrm{C}$ was observed to interrupt TMZ-induced autophagy (Fig. 2). In the current study, compound $\mathrm{C}$ was found to augment TMZ-induced DNA damage, as indicated by increased $\gamma \mathrm{H} 2 \mathrm{AX}$ detected in the $\mathrm{TMZ}+$ compound $\mathrm{C}$ group compared with the control, TMZ and compound $\mathrm{C}$ groups in U87MG and U251 cells (Fig. 4A). MTT analysis also showed that the TMZ + compound C 
$\mathbf{A}$

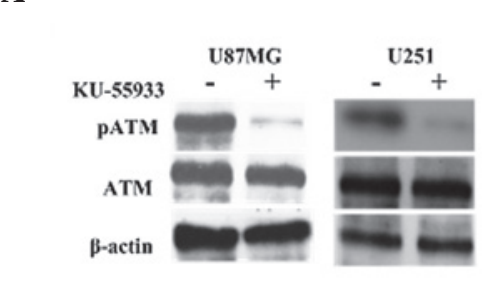

U87MG
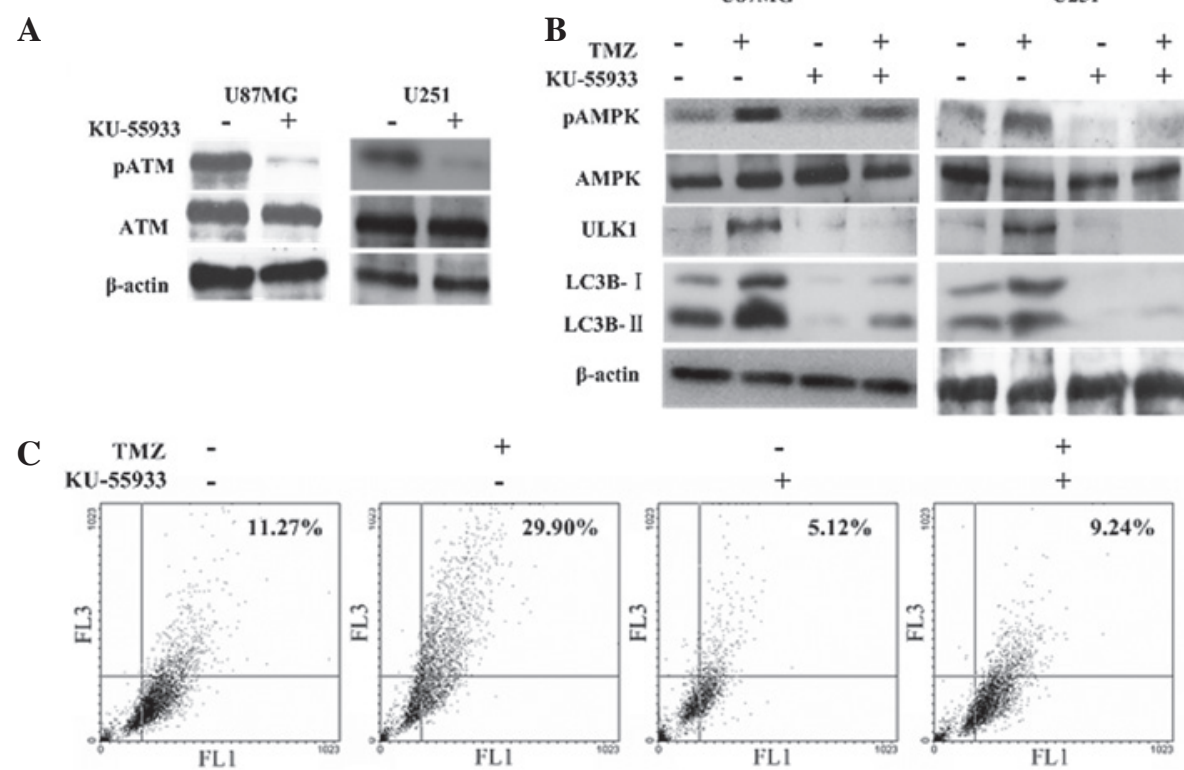

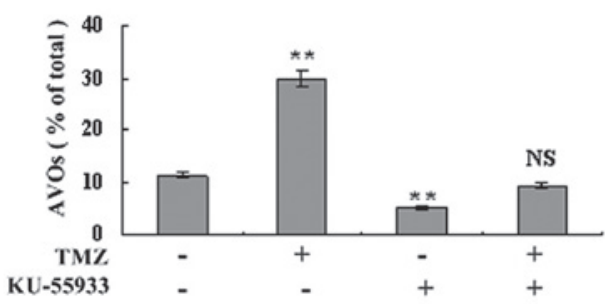

Figure 3. TMZ-induced autophagy and AMPK-ULK1 activation are ATM dependent. (A) U87MG and U251 glioma cells were treated with $10 \mu$ M KU-55933 or vehicle. Following $72 \mathrm{~h}$, cells were harvested and analyzed by western blot analysis to determine the effect of KU-55933 on ATM (Ser1981) phosphorylation. $\beta$-actin was used as a loading control. (B) U87MG and U251 cells were divided into four groups, and treated with vehicle, TMZ (100 $\mu \mathrm{M}), \mathrm{KU}-55933(10 \mu \mathrm{M})$ and TMZ $(100 \mu \mathrm{M})+\mathrm{KU}-55933(10 \mu \mathrm{M})$ for $72 \mathrm{~h}$, respectively. Subsequently, cells were collected and subjected to western blot analysis with antibodies against phosphorylated AMPK $\alpha$ (Thr172), AMPK $\alpha$, phosphorylated ULK1 (Ser467) and LC3B. $\beta$-actin was used as a loading control. (C) U87MG cells were treated with vehicle, TMZ $(100 \mu \mathrm{M})$ and/or KU-55933 $(10 \mu \mathrm{M})$ as indicated for $72 \mathrm{~h}$. Following drug treatment, acridine orange was added at a final concentration of $1 \mu \mathrm{g} / \mathrm{ml}$ for a period of $15 \mathrm{~min}$. Next, cells were collected and AVOs were detected with flow cytometry. A total of 5,000 ungated events were analyzed. Data are shown as the mean \pm standard deviation. ${ }^{*} \mathrm{P}<0.05$ and ${ }^{* *} \mathrm{P}<0.01$, vs. the control groups, $\mathrm{n}=3$ for each group. NS not statistically significant; TMZ, temozolomide; AVOs, acidic vesicular organelles; AMPK, adenosine monophosphate-activated protein kinase; ATM, ataxia-telangiectasia mutated.

A

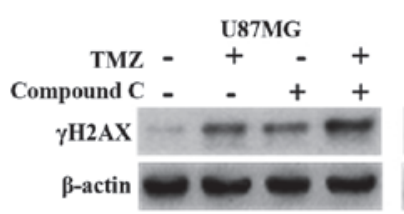

C

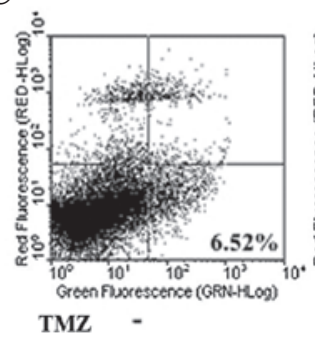

Compound C -

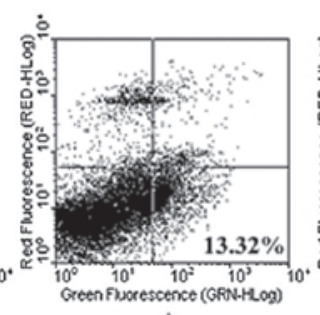

$+$

Figure 4. Inhibition of AMPK with compound C augments the cytotoxicity of TMZ. (A) U87MG and U251 cells were treated with vehicle, TMZ (100 $\mu$ M), compound $\mathrm{C}(5 \mu \mathrm{M})$ or TMZ $(100 \mu \mathrm{M})+$ compound $\mathrm{C}(5 \mu \mathrm{M})$ for $72 \mathrm{~h}$. Next, cells were collected and subjected to western blot analysis to detect the expression of $\gamma \mathrm{H} 2 \mathrm{AX}$. $\beta$-actin was used as a loading control. (B) U87MG and U251 cells were seeded at a density of 3,000 cells/well in 96-well microplates and treated with vehicle, TMZ $(100 \mu \mathrm{M})$, compound C $(5 \mu \mathrm{M})$ or TMZ $(100 \mu \mathrm{M})+$ compound $\mathrm{C}(5 \mu \mathrm{M})$ for $72 \mathrm{~h}$. Following culture, MTT assays were performed to assess the cell viability ( $\mathrm{n}=6$ for each group). (C) U87MG cells were treated with vehicle, TMZ $(100 \mu \mathrm{M})$, compound C (5 $\mu \mathrm{M})$ or TMZ (100 $\mu \mathrm{M})+\mathrm{compound} \mathrm{C}$ $(5 \mu \mathrm{M})$ for $72 \mathrm{~h}$. Subsequently, cells were collected and apoptosis was detected with Annexin-V-fluorescein isothiocyanate/propidium iodide double staining ( $\mathrm{n}=3$ for each group). Data are shown as the mean \pm standard deviation. " $\mathrm{P}<0.05$ and ${ }^{* *} \mathrm{P}<0.01$, vs. control group. AMPK, adenosine monophosphate-activated protein kinase; TMZ, temozolomide. 
group exhibited slower growth compared with that of the other groups (Fig. 4B). Next, the level of apoptosis in each group was assessed with Annexin V-FITC/PI double staining. The levels of apoptotic cells in the control, TMZ, compound $\mathrm{C}$ and TMZ + compound $\mathrm{C}$ groups were observed to be 6.52 , 13.32, 10.69 and $25.02 \%$, respectively (Fig. 4C). These results indicated that inhibition of AMPK augmented the cytotoxicity of TMZ by interrupting autophagy.

\section{Discussion}

Autophagy is a highly conserved catabolic process in which cells self-digest organelles and other macromolecules via the autophagosome. Autophagy ameliorates the negative effects of dysregulated cellular metabolism, allowing a steady supply of nutrients and removal of damaged organelles (20-22). TMZ induces an autophagy-associated adenosine triphosphate (ATP) surge through the degradation of cellular proteins and organelles, which maintains cellular homeostasis and survival. In addition, inhibition of autophagy augments TMZ-induced apoptosis $(12,23)$. The current results also showed that $100 \mu \mathrm{M}$ TMZ treatment induced autophagy in U87MG and U251 glioma cells. However, the molecular mechanisms by which TMZ induces autophagy remain largely unknown.

AMPK is a conserved sensor of intracellular energy, which is activated in response to low nutrient availability and cellular stress, and is involved in the initiation of autophagosome formation by interacting with mammalian autophagy-initiating kinase ULK1 $(18,19)$. The current results showed that TMZ treatment led to significant AMPK phosphorylation and ULK1 activation during the process of autopahgy. When AMPK phosphorylation was inhibited by compound $\mathrm{C}$, TMZ-induced autophagy was significantly interrupted as indicated by a decrease in LC3B cleavage. Next, the mechanism of AMPK-ULK1 pathway activation was investigated.

ATM kinase forms a central node in the DNA damage response phosphorylation cascade by contributing to the initiation, amplification and transmission of the DNA damage signal to downstream substrates $(7,24)$. The results indicated that AMPK-ULK1 pathways were one of these downstream pathways. TMZ failed to induce AMPK-ULK1 activation following KU-55933 treatment, which led to a decrease of LC3B cleavage and AVO formation. Thus, TMZ-induced DNA foci recruit and activate ATM kinase, which in turn evokes phosphorylation of AMPK-ULK1 and subsequently elicits autophagy. Thus, glioma cells may supply steady nutrients and energy for DNA damage repair or other cellular processes.

Based on this hypothesis, interruption of ATM-AMPK-ULK1 pathways results in autophagy inhibition and augment TMZ cytotoxicity. To examine this hypothesis, autophagy was interrupted with the AMPK inhibitor compound $\mathrm{C}$, and AMPK inhibition was found to augment TMZ cytotoxicity as observed by impaired cell viability, an increase of $\gamma \mathrm{H} 2 \mathrm{AX}$-marked DSBs and elevated numbers of apoptotic U87MG cells. We hypothesize that AMPK inhibition interrupts the cytoprotective process of autophagy, which results in augmentation of the TMZ cytotoxic effect and promotes glioma cell death under apoptotic stress. These results suggest that glioma chemoresistance may be overwhelmed by targeting AMPK, particularly for MGMT-negative patients.
In conclusion, TMZ treatment induces autophagy through ATM-AMPK-ULK1 pathways, and AMPK inhibition augments TMZ cytotoxicity. The current results suggest that AMPK may be a treatment target to overwhelm TMZ chemoresistance.

\section{Acknowledgements}

The authors would like to acknowledge financial supports from the China Postdoctoral Science Foundation (grant no. 2012M512182), the Guangdong Natural Science Foundation (grant no. S2012040006588) and the Guangzhou Science and Technology Project (grant no. 201300000150).

\section{References}

1. Schwartzbaum JA, Fisher JL, Aldape KD and Wrensch M: Epidemiology and molecular pathology of glioma. Nat Clin Pract Neurol 2: 494-503, 2006.

2. Stupp R, Mason WP, van den Bent MJ, et al: Radiotherapy plus concomitant and adjuvant temozolomide for glioblastoma. N Engl J Med 352: 987-996, 2005.

3. Caporali S, Falcinelli S, Starace G, et al: DNA damage induced by temozolomide signals to both ATM and ATR: role of the mismatch repair system. Mol Pharmacol 66: 478-491, 2004.

4. Zhang J, Stevens MF and Bradshaw TD: Temozolomide: mechanisms of action, repair and resistance. Curr Mol Pharmacol 5: 102-114, 2012.

5. Wu J, Zhang X, Zhang L, et al: Skp2 E3 ligase integrates ATM activation and homologous recombination repair by ubiquitinating NBS1. Mol Cell 46: 351-361, 2012.

6. Andegeko Y, Moyal L, Mittelman L, Tsarfaty I, Shiloh Y and Rotman G: Nuclear retention of ATM at sites of DNA double strand breaks. J Biol Chem 276: 38224-38230, 2001.

7. Shiloh Y and Ziv Y: The ATM protein kinase: regulating the cellular response to genotoxic stress, and more. Nat Rev Mol Cell Biol 14: 197-210, 2013

8. Jiang G, Li LT, Xin Y, Zhang L, Liu YQ and Zheng JN: Strategies to improve the killing of tumors using temozolomide: targeting the DNA repair protein MGMT. Curr Med Chem 19: 3886-3892, 2012.

9. Skiriute D, Vaitkiene P, Saferis V, et al: MGMT, GATA6, CD81, DR4, and CASP8 gene promoter methylation in glioblastoma. BMC Cancer 12: 218, 2012.

10. Felsberg J, Thon N, Eigenbrod S, et al: Promoter methylation and expression of MGMT and the DNA mismatch repair genes MLH1, MSH2, MSH6 and PMS2 in paired primary and recurrent glioblastomas. Int J Cancer 129: 659-670, 2011.

11. Natsumeda M, Aoki H, Miyahara H, et al: Induction of autophagy in temozolomide treated malignant gliomas. Neuropathology 31 : 486-493, 2011

12. Lin CJ, Lee CC, Shih YL, et al: Inhibition of mitochondria- and endoplasmic reticulum stress-mediated autophagy augments temozolomide-induced apoptosis in glioma cells. PLoS One 7: e38706, 2012.

13. Kabeya Y, Mizushima N, Yamamoto A, Oshitani-Okamoto S, Ohsumi Y and Yoshimori T: LC3, GABARAP and GATE16 localize to autophagosomal membrane depending on form-II formation. J Cell Sci 117: 2805-2812, 2004.

14. Wu J, Dang Y, Su W, et al: Molecular cloning and characterization of rat LC3A and LC3B - two novel markers of autophagosome. Biochem Biophys Res Commun 339: 437-442, 2006.

15. Murrow L and Debnath J: Autophagy as a stress-response and quality-control mechanism: implications for cell injury and human disease. Annu Rev Pathol 8: 105-137, 2013.

16. Paglin S, Hollister T, Delohery T, et al: A novel response of cancer cells to radiation involves autophagy and formation of acidic vesicles. Cancer Res 61: 439-444, 2001.

17. Graf MR, Jia W and Loria RM: The neuro-steroid, 3beta androstene 17alpha diol exhibits potent cytotoxic effects on human malignant glioma and lymphoma cells through different programmed cell death pathways. Br J Cancer 97: 619-627, 2007.

18. Wong PM, Puente C, Ganley IG and Jiang X: The ULK1 complex: sensing nutrient signals for autophagy activation. Autophagy 9: 124-137, 2013. 
19. Sanchez AM, Csibi A, Raibon A, et al: AMPK promotes skeletal muscle autophagy through activation of forkhead FoxO3a and interaction with Ulk1. J Cell Biochem 113: 695-710, 2012.

20. Leone RD and Amaravadi RK: Autophagy: a targetable linchpin of cancer cell metabolism. Trends Endocrinol Metab 24: 209-217, 2013.

21. Cheong H, Lu C, Lindsten T and Thompson CB: Therapeutic targets in cancer cell metabolism and autophagy. Nat Biotechnol 30: 671-678, 2012.
22. Choi AM, Ryter SW and Levine B: Autophagy in human health and disease. N Engl J Med 368: 651-662, 2013.

23. Katayama M, Kawaguchi T, Berger MS and Pieper RO: DNA damaging agent-induced autophagy produces a cytoprotective adenosine triphosphate surge in malignant glioma cells. Cell Death Differ 14: 548-558, 2007.

24. Marinoglou K: The role of the DNA damage response kinase ataxia telangiectasia mutated in neuroprotection. Yale J Biol Med 85: 469-480, 2012 